\title{
AVALIAÇÃO EPIDEMIOLÓGICA DE PACIENTES DIAGNOSTICADOS COM DEGENERAÇÃO MACULAR RELACIONADA À IDADE NO HOSPITAL DE OLHOS DO TOCANTINS
}

\author{
EPIDEMIOLOGICAL EVALUATION OF DIAGNOSTIC PATIENTS WITH \\ MACULAR DEGENERATION RELATED TO AGE IN THE EYE \\ HOSPITAL OF TOCANTINS
}

\author{
Érica Parreão Cunha ${ }^{1}$ \\ Bruna Cardoso de Melo ${ }^{2}$ \\ João Carlos Diniz Arraes ${ }^{3}$ \\ Rejanne Lima Arruda ${ }^{4}$
}

RESUMO: OBJETIVO: Avaliar a epidemiologia de pacientes diagnosticados com degeneração macular relacionada à idade atendidos no Hospital de Olhos do Tocantins, da cidade de Araguaína no ano de 2020. MÉTODO: Trata-se de um estudo, descritivo, transversal e analítico com uma abordagem quantitativa. A pesquisa ocorreu no município de Araguaína (Tocantins) no Hospital dos Olhos do Tocantins. A amostra é composta por 103 pacientes atendidos no período do ano 2020 que apresentaram Degeneração Macular Relacionada a Idade(DMRI) e que se adequaram aos critérios de inclusão e exclusão. Foram coletadas informações por meio de prontuários e os dados tabulados pelo Microsoft Excel. $\mathrm{O}$ estudo seguiu em conformidade com a Resolução $n^{\circ} 466 / 12$ do Conselho Nacional de Saúde. RESULTADOS: Entre os 103 amostrados, a pesquisa mostrou que o público mais acometido é o entre a faixa etária de 60 e 79 anos de idade, do sexo feminino. No que se refere a história de Hipertensão Arterial Sistêmica foi maior o número de pacientes diagnosticados com a comorbidade. Quanto a forma clínica da doença houveram mais casos de DMRI na sua variante seca, dentre esses houve

\footnotetext{
${ }^{1}$ Acadêmica do curso de medicina - Centro Universitário Tocantinense Presidente Antônio Carlos. Araguaína, Tocantins.

${ }^{2}$ Acadêmica do curso de medicina - Centro Universitário Tocantinense Presidente Antônio Carlos. Araguaína, Tocantins.

${ }^{3}$ Professor da Universidade Federal do Tocantins; Coordenador da Residência Médica em Oftalmologia do HO/UFT/HDT; Doutorado pela UFG Diretor e Médico-Oftalmologista do Hospital de Olhos do Tocantins.

${ }^{4}$ Professora Doutora do Centro Universitário Tocantinense Presidente Antônio Carlos. Araguaína, Tocantins.
} 
predominância de boa acuidade visual. Na forma úmida houve baixa visual mais significativa e na avançada mais casos de baixa acuidade visual, sendo essa considerada cegueira legal. CONCLUSÃO: Os resultados encontrados no presente estudo estão de acordo com a literatura. Dessa forma, a detecção precoce de pacientes com DMRI torna-se fundamental para evitar a sua evolução para formas clínicas mais graves que cursam com cegueira legal.

Palavras chave: Retinopatias. Saúde pública. Envelhecimento. Cegueira.

ABSTRACT: OBJECTIVE: To evaluate the epidemiology of patients diagnosed with age- related macular degeneration at Eye Hospital of Tocantins. METHOD: It is a study descriptive, transversal and analytical with a quantitative approach. The research takes place in the municipality of Araguaina (Tocantins) at Eye Hospital of Tocantins. Epidemiological data were collected from medical records. The sample consists of 103 patients seen in the period of the year 2020, which added to AgeRelated Macular Degeneration (AMD) and which met the inclusion and exclusion criteria. Information was collected through medical records and data tabulated by Microsoft Excel. The study followed in compliance with Resolution No. 466/12 of the National Health Council. RESULTS: Among the 103 sampled, the research showed that the public most affected is the one between the age group of 60 and 79 years old, female. Regarding the history of Systemic Arterial Hypertension, the number of patients diagnosed with the comorbidity was higher. As for the clinical form of the disease, there were more cases of AMD in its dry variant, among which there was a predominance of good visual acuity. In the wet form, there was a more significant visual loss and in the advanced case, more cases of low visual acuity, which was considered legal blindness. CONCLUSION: The results found in thepresent study are in accordance with the literature. Thus, the early detection of patients with AMD is essential to prevent their progression to more severe clinical forms that evolve into legal blindness.

Keywords: Retinopathies. Public health. Aging. Blindness. 


\section{INTRODUÇÃO}

A Degeneração Macular Relacionada à Idade (DMRI) é uma doença causada por danos à mácula, uma região da retina, responsável pela visão no centro do campo visual. Embora possua etiologia indefinida, há componentes multifatoriais. $A$ presença da doença significa perda progressiva da visão e é uma das principais causas de cegueira em países desenvolvidos(ALTEMANI; AMSTELDAN, 2016).

A mácula é uma região amarelada encontrada na retina posterior no lado temporal. Essacontém células fotorreceptoras, os cones, o citoplasma das células de Müller, células ganglionares e células da camada nuclear interna, sendo muito importante na visão de cores, na clareza e definição da imagem. Mensura-se que cerca de $90 \%$ da visão é transmitida ao cérebropela mácula (QUEIROZ et al, 2010).

Predominantemente existem duas formas de DMRI, a seca (não exsudativa), mais comum, onde ocorre a formação de drusas amareladas sob o epitélio pigmentar da retina (EPR) e na membrana de Bruch, causando atrofia do EPR, que afeta a atividade dos fotorreceptores econsequentemente levam a perda de visão e a úmida (exsudativa), onde há neovascularização proveniente dos coriocapilares que penetra a membrana de Bruch e o EPR podendo causar hemorragias, também atingindo fotorreceptores e reduzindo a acuidade visual (YANOFF; CAMERON, 2014).

Conforme o AGE-RELATED EYES DISEASE STUDY (2001), há ainda a DMRI avançada, esta se dá quando na forma seca acontece atrofia geográfica ou no caso da úmida haja formação da membrana vascular subretiniana (MNVSR).

Um fator importante do grupo seca, embora não totalmente explicado, é a formação dedrusas, essas são depósitos extracelulares de localização variável. Em sua composição é provável que tenha materiais sintetizados pelas células do EPR, apoliproteínas $B$ e $E$, imunoglobulinas, fibrinogênio e vitronectina. As drusas se dividem em duras e moles, sendo as duras caracterizadas por formato arredondado bem definido, hialinidade, eosinofilia e formação de elevações na superfície da membrana de Bruch (MB). Enquanto as moles aparentam ser indefinidas (em 
camadas de material granular mole e se depositam na lâmina basal da $M B$, deslocam o EPR da superfície interna da MB, além de interferirem na atividade da córiocapilar. Há também a ocorrência de hiperpigmentação focal do EPR e atrofia geográfica (AG), a primeira se apresenta como pontos de bloqueio da fluorescência e está associado a maior progressão para a forma úmida da doença. Já a AG há apoptose de células do EPR com consequente diminuição dos fotorreceptores, sendo essa a principal causa de cegueira legal entre a forma seca. (ÁVILA et al., 2011).

No que tange a forma úmida, há uma associação com a neovascularização coroidal (NVC), que é o crescimento a partir do coriocapilar de novos vasos que penetrarão a MB. Quando essa neovascularização é restrita ao EPR, classifica-se como tipo um, caso haja crescimento para o espaço sub-retiniano, é conhecida como tipo dois e se houver neovascularização nas camadas internas da retina classifica-se como tipo três. Além dessas, alguns fatores podem influenciar a NVC, como o fator de crescimento endotelial vascular (VEGF), componentes inflamatórios e a integridade da MB e do EPR. (SALMON, 2020).

Segundo estudo feito por Rim (2012), a idade avançada, o tabagismo, a cirurgia préviade catarata e os antecedentes familiares de DMRI são, dentre todos, os fatores de maior risco para a progressão da doença.

Para o diagnóstico inicial de DMRI, além da clínica é recomendado a fundoscopia comas pupilas dilatadas. Para a avaliação da integridade funcional, do fluxo sanguíneo da retina e coroidéia e detalhes do epitélio pigmentar é usada a angiografia fluoresceínica, que é um exame que utiliza o contraste fluoresceína. Frente a forma exsudativa o diagnóstico é realizado através da angiografia fluoresceínica e/ou Tomografia de Coerência Óptica (OCT) - procedimento que usa luz para obter e criar uma imagem da retina e do disco óptico com a técnica chamada interferometria de baixa coerência para medidas ópticas- o mesmo é usado tanto no diagnóstico quando no acompanhamento da doença. Entretanto, quando usados separadamente, há uma pequena porcentagem de que não detectem casos leves da doença (PARANHOS et al, 2013).

O tratamento da DMRI exsudativa atualmente é realizado com aplicação de injeções intravítrea de anti -VEGF (Fator de crescimento endotelial vascular), o qual 
permite uma importante melhora no prognóstico visual destes pacientes que até a última década julgava-se intratáveis (KHANNA et al, 2019).

Os principais anti-VEGFs utilizados na atualidade são bevacizumabe, ranibizumabe e aflibercept, os quais apresentam eficácia semelhante (PARK; SUN; LEE, 2016).

Segundo o último censo demográfico feito pelo IBGE (2010), a população do Tocantins corresponde a 1.383 .445 habitantes e cerca de $14 \%$ possui idade acima dos 50 anos, sendo essaporcentagem um indicativo importante da população sujeita ao desenvolvimento de DMRI. Deste modo, torna-se importante avaliar a prevalência de DMRI nesses habitantes e sobretudo analisar os fatores epidemiológicos desta patologia, afim de definir um perfil para o acometimento de pacientes, e assim buscar formas de prevenção e consequentemente a diminuição do número de casos avançados da doença, além de melhorar a qualidade vida, proporcionando um envelhecimento mais saudável.

Diante do exposto, o estudo objetiva-se em avaliar a epidemiologia de pacientes diagnosticados com degeneração macular relacionada à idade atendidos no Hospital de Olhos do Tocantins, da cidade de Araguaína no ano de 2020.

\section{METODOLOGIA}

Foi realizado uma pesquisa do tipo descritiva, transversal e analítica utilizando os prontuários de pacientes que apresentam Degeneração Macular Relacionada a Idade (DMRI) assistidos peloHospital de Olhos do Tocantins (H.O.T) no ano de 2020, incluindo pacientes já diagnosticados com a doença, excluindo aqueles não diagnosticados ou os quais o preenchimento do prontuário foi incompleto em relação ao que foi avaliado no estudo. O acesso ao prontuário foi realizado por meio do Sistema de Gestão Hospitalar- Gemmius ${ }^{\circledR}$.

Tais dados quantitativos só foram coletados após submissão e aprovação, através da Plataforma Brasil, ao Comitê de Ética em Pesquisa (CEP) do Centro Universitário Tocantinense Presidente Antônio Carlos cujo número do parecer de 
aprovação é 4.435 .575 (ANEXO 1). Foram coletadas informações por meio de prontuários e os dados tabulados pelo Microsoft Excel.

Dentre as informações coletadas, há idade (40-59 anos, 60-79 anos e $\geq 80$ anos), sexo, presença ou não de hipertensão arterial, tipo de DMRI (seca, úmida e avançada) e acuidade visual. Quanto a acuidade visual, foi considerada a classificação de boa sendo a acuidade visual até 20/40, moderada de 20/50 até 20/400 também chamada de visão subnormal e baixa acima de 20/400, que é considerada cegueira legal (OTTAIANO et al., 2019).

O estudo será conduzido em conformidade a Resolução $n^{\circ} 466 / 12$ do Conselho Nacional de Saúde, cumprindo os aspectos éticos e legais necessários para o desenvolvimento de pesquisas envolvendo seres humanos (BRASIL,2013).

\section{RESULTADOS}

Dos 103 pacientes analisados, 56 (54,37\%) eram do sexo feminino, enquanto $47(45,63 \%)$ eram do sexo masculino. No que tange a idade, $13(12,62 \%)$ estão entre a faixa etária de 40-59 anos, $62(60,2 \%)$ possui de 60-79 anos, enquanto 28 $(27,18 \%)$ tem 80 anos ou mais. No que diz respeito a Hipertensão Arterial Sistêmica (HAS) $53(51,43 \%)$ possuíam esse antecedente patológico pregresso e 50 (48,54\%) negaram. Esses dados foram ilustrados na Tabela 1. 
Tabela 1 - Sexo, idade, e diagnóstico de HAS em pacientes diagnosticados com DMRI no anode 2020 no H.O do Tocantins, da cidade de Araguaína.

\begin{tabular}{|c|c|c|}
\hline Variáveis & $\mathbf{n}$ & $\%$ \\
\hline \multicolumn{3}{|l|}{$\overline{\text { Sexo }}$} \\
\hline Feminino & 56 & 54,37 \\
\hline Masculina & 47 & 45,63 \\
\hline \multicolumn{3}{|l|}{ Idade (anos completos) } \\
\hline $40-59$ & 13 & 12,62 \\
\hline $60-79$ & 62 & 60,2 \\
\hline$\geq 80$ anos & 28 & 27,18 \\
\hline \multicolumn{3}{|l|}{ Diagnóstico de HAS } \\
\hline Sim & 53 & 51,46 \\
\hline Não & 50 & 48,54 \\
\hline
\end{tabular}

Fonte: Elaboração Própria.

Dos 206 olhos avaliados, 137(66,50\%) foram diagnosticados com DMRI seca, $42(20,39 \%)$ entraram na classificação de DMRI úmida e $27(13,11 \%)$ desses ficaram no estágio considerado avançado da doença. Conforme a Tabela 2.

Tabela 2 - Classificação dos tipos de DMRI nos olhos avaliados.

TIPOS DE NÚMERO DE OLHOS DIAGNÓSTICADOS COM DMRI

\begin{tabular}{ccc}
\hline DMRI & $\mathbf{N}$ & $\%$ \\
\hline Seca & 137 & 66,50 \\
Úmida & 42 & 20,39 \\
Avançada & 27 & 13,11 \\
TOTAL & 206 & 100 \\
\hline
\end{tabular}

Fonte: Elaboração Própria.

Se tratando da forma seca $66(48,18 \%)$ obtiveram boa acuidade visual, 54 $(39,42 \%)$ moderada e $17(12,40 \%)$ baixa. Na forma úmida, 17 (40,48\%) obtiveram boa acuidade visual, $11(26,17 \%)$ moderada e $14(33,33 \%)$ baixa. Nos classificados como avançados $2(7,41 \%)$ tinham uma visão boa, 2 (7,41\%) moderada e 23 $(85,19 \%)$ baixa. Tal comparação está expressana Tabela 3. 
Tabela 3 - Relação entre classificação da DMRI com acuidade visual.

\begin{tabular}{|c|c|c|c|c|c|c|}
\hline \multirow{2}{*}{$\begin{array}{c}\text { TIPO DE } \\
\text { DMRI }\end{array}$} & \multicolumn{5}{|c|}{ ACUIDADE VISUAL } \\
\cline { 2 - 7 } & \multicolumn{2}{|c|}{ BOA $^{*}$} & \multicolumn{2}{c|}{ MODERADA $^{* *}$} & \multicolumn{2}{c|}{ BAIXA $^{\text {*** }}$} \\
\cline { 2 - 7 } & $\mathbf{n}$ & $\mathbf{\%}$ & $\mathbf{n}$ & $\mathbf{\%}$ & $\mathbf{n}$ & $\%$ \\
\hline Seca & 66 & 48,18 & 54 & 39,42 & 17 & 12,40 \\
\hline Úmida & 17 & 40,48 & 11 & 26,19 & 14 & 33,33 \\
\hline Avançada & 2 & 7,41 & 2 & 7,41 & 23 & 85,18 \\
\hline
\end{tabular}

${ }^{*}$ BOA: até 20/40; ${ }^{* *}$ MODERADA 20/50 até 20/400; ${ }^{* * *}$ BAIXA: > 20/400.

Fonte: Elaboração Própria.

\section{DISCUSSÃO}

Como já mencionado na titulação da doença, a idade é o principal fator de risco para Degeneração Macular Relacionada a Idade. No presente estudo, a faixa etária com maior número de pacientes diagnosticados foi a de 60 a 79 anos, contrapondo os resultados obtidos por Ferro (2018) no qual, o percentual de pacientes acometidos pela DMRI é maior a partir dos 80 anos. Pode-se justificar os resultados obtidos no presente estudo devido ao fato dos pacientesdentro desta faixa etária de 60-79 anos terem uma vida social mais ativa, e sentirem necessidade em manter uma boa acuidade visual, além do fato da perspectiva de vida dos habitantes da região ser em torno de 74 anos, segundo dados do IBGE (2020).

Dentre os fatores de risco para DMRI descritos na literatura como suspeitos, sendo sua influência incerta, estão: a cor azul da íris, a exposição solar e o sexo feminino (SALMON, 2020). Entretanto, como este estudo tinha como fonte de dados os prontuários dos pacientes e informações como a cor da íris e a exposição solar não continham nos mesmos, dos fatores suspeitos pôde-se avaliar o sexo dos pacientes. Dessa forma, apesar de ter sido por uma pequena diferença $(8,74 \%)$ a porcentagem de pacientes do sexo feminino $(54,37 \%)$, foi maior do que a do masculino $(45,63 \%)$. 
Em relação a forma seca, tipo de DMRI mais prevalente $(66,5 \%)$, uma porcentagem considerável dos olhos $(48,18 \%)$ apresentou uma boa acuidade visual (melhor ou igual à 20/40),já dos que apresentaram a forma úmida (42 olhos), 59,52\% deles obtiveram uma baixa visual mais significativa, pior que 20/40. Conforme esperado, os olhos com o estágio avançado da doença (cicatriz disciforme ou atrofia geográfica), foram os que possuíram pior acuidade visual sendo 85,19\% considerados com cegueira legal (pior ou igual a 20/400). Segundo a literatura, a perda visual da forma úmida geralmente é mais rápida e mais acentuada que a da forma seca, entretanto no presente estudo, alguns olhos com DMRI exsudativa se encontram com boa acuidade visual, e isso deve-se ao fato de que os pacientes em questão estão em tratamento comterapia de antiangiogênica intravítreo.

Segundo Torres (2009) a hipertensão arterial sistêmica está entre os fatores de risco para DMRI por influenciar na circulação coroidal, devido ao seu potencial isquêmico. Contudo, a diferença entre os pacientes com DMRI que possuíam antecedentes patológicos de HAS e os que não o tinham, não foi relevante, uma vez que foi de $2,92 \%$.

Além dos fatores de risco avaliados no presente estudo, existem outros que possuem uma forte ligação com a doença, sendo eles: tabagismo, obesidade, hereditariedade e cor da pele. Suspeita-se que o tabaco reduz a concentração de antioxidantes no plasma, interferindo de modo indireto no metabolismo coriorretiniano, além de estimular a formação aterosclerótica nos vasos sanguíneos coroidais (TORRES, 2009). No entanto, outro estudo, que avalia a prevalência e os fatores de risco, não encontra nenhuma correlação estatística entre os pacientes com DMRI e o tabagismo (SANTOS, 2005). Além disso, outros fatores também são descritos na literatura, como a dieta com alta ingestão de gorduras e a obesidade. Também é citado a história familiar, uma vez que já é comprovada a associação, seja de proteção ou risco, da DMRI com os cromossomos 1q32 para o fator $\mathrm{H}$ do complemento (CFH), o gene hemicentin em 1q24-25 e o gene ABCR no cromossomo 1p (SALMON, 2020). Contudo por não conseguir obter essas informações em todos os prontuários analisados, a comparação ficou inviável. 


\section{CONCLUSÃO}

Os resultados encontrados no presente estudo estão de acordo com a literatura, sendo eles: O predomínio da forma seca da doença, a relação da doença com a idade, com a hipertensão arterial sistêmica e com o sexo feminino. Contudo, quanto a relação da DMRI com outros fatores como exposição solar, tabagismo e histórico familiar, não foi possível ser avaliada devido a obtenção das informações ter sido a partir de dados secundários. Por fim, nota-se uma piora da acuidade visual em pacientes classificados na forma úmida ou avançada da doença. Dessa forma, a detecção precoce de pacientes com DMRI torna-se fundamental para evitar a evolução dessa patologia para formas clínicas mais graves como a forma úmida e avançada, que cursam com pior acuidade visual e que podem resultar em sequelas como cegueira legal, que afetam significativamente a qualidade de vida do paciente.

\section{REFERÊNCIAS BIBLIOGRÁFICAS}

Age-Related Eye Disease Study Research Group. A Randomized, Placebo-Controlled, Clinical Trial of High-Dose Supplementation With Vitamins C and E, Beta Carotene, and Zinc for AgeRelated Macular Degeneration and Vision Loss: AREDS Report No. 8. Arch Ophthalmol, American Medical Association, 1 out. 2001.

ALTEMANI, Albina Messias; AMSTALDEN, Eliane Maria. Patologia Otorrinolaringológica. In: FILHO, Geraldo Brasileiro. Bogliolo Patologia. 9. ed. Rio de Janeiro RJ: Guanabara Koogan, 2016. cap. 30, ISBN 9788527729833. ÁVILA, Marcos et al. Retina. In: DANTAS, Adalmir Morterá. Essencial em Oftalmologia. 1. ed. Rio de Janeiro RJ: Guanabara Koogan, 2011. cap. 14, p. 484-532. ISBN 9788570064691.

BRASIL. Resolução $n^{\circ} 466$, de 12 de dezembro de 2012. Dispõe sobre diretrizes e normas regulamentadoras de pesquisas envolvendo seres humanos. Diário Oficial [da] República Federativa do Brasil, Brasília, DF, 13 jun. 2013. Disponível em: <Disponível em: http://bit.ly/1mTMIS3 > Acesso em: 14 jun. 2020.

FERRO, Ana Paula de Costa. Degeneração macular relacionada à idade: prevalência e perfil dos pacientes. 2018.

IBGE - Instituto Brasileiro de Geografia e Estatística. Censo Demográfico. 2010.

IBGE - Instituto Brasileiro de Geografia e Estatística. Projeção da população 2010-2060. 2020.

KHANNA, Saira et al. Current and Upcoming anti-VEGF therapies and dosing strategies for the 
treatment of neovascular AMD: a comparative review. BMJ Open Ophthalmology, [s. I.],11 dez. 2019. Disponível em: http://bmjophth.bmj.com/. Acesso em: 4 jun. 2020.

OTTAIANO, José Augusto Alves; ÁVILA, Marcos Pereira de; UMBELINO, Cristiano Caixeta; TALEB, Alexandre Chater. Afinal, o que é cegueira? E baixa visão? As condições de saúde ocular no Brasil, São Paulo, SP, ed. 1, 2019.

PARANHOS FRL, COSTA RA, MEIRELLES R, SIMÕES R. Degeneração Macular Relacionada à idade. Rev. Assoc. Med. Bras., São Paulo, v. 59, n. 2, p. 106-111, Apr. 2013.

PARK, Dae Hyun; SUN, Hae Jung; LEE, Sung Jin. A comparison of responses to intravitreal bevacizumab, ranibizumab, or aflibercept injections for neovasculae age-related macular degeneration. Int Ophthalmol, Seoul, South Korea, 8 nov. 2016.

QUEIROZ, Joaquim Marinho de et al. Degeneração macular relacionada à idade: considerações histopatológicas. Rev. Bras. De Oftalmologia, [s. I.], v. 69, ed. 6, p. 400-406, 20 nov. 2010.

RIM, Priscila Hae Hyun. Degeneração Macular Relacionada à Idade: Estudo dos fatores de risco em uma população brasileira. Orientador: Antonia Paula Marques de Faria. 2012. 143 f. Tese (Doutorado em ciências médicas) - Faculdade de Ciências Médicas, Universidade de Campinas, Campinas SP, 2012.

SALMON, John F. Acquired Macular Disorders. In: SALMON, John F. Kanski Clinical Ophtalmology: A Systematic Approach. 9. ed. [S. I.]: Elsevier, 2020. cap. 12, p. 555-614. ISBN 9780702077111.

SANTOS, Laura Patrícia Ferreira et al. Degeneração macular relacionada à idade: prevalênciae fatores de risco em dois centros oftalmológicos de referência em Pernambuco. Arq. Bras. Oftalmol., São Paulo, v. 68, n. 2, p. 229-233, Apr. 2005.

TORRES, Rogil José de Almeida et al. Fatores modificáveis da degeneração macular relacionada à idade. Arq. Bras. Oftalmol., São Paulo, v. 72, n. 3, p. 406-412, jun. 2009.

YANOFF, Myron; CAMERON, Douglas. Doenças do sistema visual. In: GOLDMAN, Lee et al. Cecil Medicina. 24. ed. Rio de Janeiro RJ: Elsevier, 2014. v. 1, cap. 431, ISBN 9788535246772.20. 